\title{
Investigating sound through vibrations
}

\author{
Aminat Ajose ${ }^{1}$ \\ ${ }^{1}$ Journal of Conceptual and Applied STEM Education
}

August 14, 2020

\section{Introduction}

In this hands on lesson, learners will explore sound waves through the concept of vibrations. They will investigate how sound travels through string and use this knowledge to design their own telephone using a paper cup and a string.

\section{Lesson Duration: 50 minutes}

\section{Lesson Objectives}

1. Learners should be able to describe how sound travels in different media.

2. Learners will work collaboratively in pairs and small groups to carry out simple investigations about sound waves.

3. Learners should use tools and materials to design and build a device that uses sound to communicate.

4. Learners should understand that technology has helped people communicate over long distances.

\section{Educational Standards}

NGSS:

Connections by topic

Physical Science: 1. Waves: Light and Sound Connections by disciplinary core ideas:

Physical Science: 1-PS4 Waves and Their Applications in Technologies for Information transfer Connections by scientific \& engineering practices 1. Asking questions \& defining problems 3. Planning and carrying out investigations 6 . Constructing explanations and designing solutions

Connections by crosscutting concepts

2. Cause and effect: Mechanism and explanation

7. Stability and change

Connections by performance expectation: 
1-PS4-1. Plan and conduct investigations to provide evidence that vibrating materials can make sound and that sound can make materials vibrate.

\section{Vocabulary}

Alexander Graham Bell: Inventor of the telephone.

Medium: A substance that sound travels through

Sound: Vibrations that travel through from their point of origin to a persons's ear

Vibration: A shaky, back and forth motion of tiny particles that form materials

Sound waves: pattern that particles make when they vibrate to create sound

\section{Integrated technology}

Watch a lesson about sound waves in http://www youtube. com/watch?v=_vYYqRVi8vY

\section{Materials}

- 2 paper cups

- 2 paper clips

- Piece of string (about 2 feet, or $30 \mathrm{~cm}$ )

- A sharpened pencil

\section{Lesson plan}

\section{ENGAGE}

Introduction

1. Gather the learners and get them thinking about the idea of communication by conducting a quick brainstorm.

"I would like you to give me some examples of how people communicate "

I will note down all responses.

2. Activate learners previous knowledge with a discussion of sound

"Can you tell me what you know about sound? What kind of things makes sound?"

3. Ask learners "Would your friend on the other side of the playground be better able to hear you through a solid or through the air?"

(I will note down all responses.) 
4. Ask the learners to put their ears to the desk and scratch the surface with their fingernail. Ask them to sit up and again scratch the desk surface.

5. Ask the following questions

What did you observe?

Did you hear a sound?

If you can hear a sound through your desk, does that mean your desk is moving?

Was there a difference in the two instances?

(I will take a few learners responses one at a time. I will also make sure they recognize that the sounds are louder with their ears to the desks.)

6. I Inform learners that

"Today we're going to explore the question: How does sound travel from one object to our ears?" We are going also going to learn more about how sound travels. We are going to model a telephone using cups and some string, analyze how sound moves through our model, and think about how the model relates to a telephone.

7. Gather answers from learners idea and experiences

"Does anyone have any thoughts or guesses?"

"Let's see if we can gather some information to help us make good guesses (hypotheses)."

\section{EXPLORE}

1. Ask learners to make a prediction(hypothesis)

"Before we begin, I want you to make a hypothesis about what you think will happen when one person whispers into one of the cups. What will happen if the string is pulled tight? What will happen if the string is lose? What will happen if the string is bent around a corner or over a table?"

2. I will then demonstrate how to make a string telephone.

"I am going to demonstrate how to build your phone. First you take two cups and poke a hole in the bottom of each one (with a pencil). Then you feed your string through each hole. Finally you tie a paperclip onto each end of the string. This way it won't come out of the cup. Once you have made your phone, you will need to work with your partner and try to figure out how to get your phone to work. Once you get it to work, you will need to explain how and why it works."

3. Pair learners into team of two and give each team of kids two paper cups, two paper clips, a sharpened pencil, and $30 \mathrm{~cm}$ string.

4. Allow learners work in team to create a string telephone using two plastic cups and one piece of string. They will work as a group to complete the experiment in order to answer the questions in their hypothesis.

5. Guide the group in discussion.

"Can you hear your partner speak to you?"

"How do you think this telephone works?"

"What is doing the vibration?" 
"What is initiating the vibration?"

"Where is the vibration ending up?"

"What could you do to make the string telephone work better?"

\section{EXPLAIN}

The focus of my discussion will be about the students thoughts on how and why the phones worked. As the teacher, I want to make sure that the idea of vibrating materials make sound and sound can make materials vibrate is well understood by my learners.

1. I will remind learners that sounds produce waves or vibrations much like the vibration of the rubber band and vocal chords. These vibrations can be transferred through solids, liquids, or gases and received on another end. They just must have the right type of receiver to interpret the sound. The most common example is our eardrum. When sound travels through a solid, it travels the same way as it does through air: in a sound wave. The sound wave actually moves the tiny particles, or molecules, that make up the solid.

2. I will remind learners of their experience with the desk to understand that sound waves sound louder when we hear them through solids.

\section{Elaborate}

1. Allow learners to watch a video to help reinforce how sound travels http://www. youtube.com/ watch?v=_vYYqRVi8vY

2. Extend the knowledge to real world application. Inform learners that

"Scientists learn about sound waves to develop technologies that help people talk to each other over long distances, like the telephone, cell phone, or the internet.

3. Guide learners to discuss other ways that vibrations and sound waves are helping us in our daily lives.

\section{EVALUATE}

1. Ask learners: What question were we trying to figure out? (how sound travels from objects to ears)

What did we learn with the desk and finger scratching experiment? What did we learn with the cup telephone?

2. What is sound? 


\section{Assessment:}

Formative:

I will observe the learners as they work. As learners are working, I circulate to make sure they can successfully create their phones. However, I don't offer input on how and why they work.

Summative: learners will turn in their hypothesis and their answers to the questions 